\title{
Spectrum Availability Aware Routing and Resource Allocation for Point-to- Multipoint Services in Mixed-Grid Optical Networks
}

\author{
Feng WANG ${ }^{\mathrm{a}}$, Qingcheng ZHU ${ }^{\mathrm{b}}$, Xiaolong $\mathrm{LI}^{\mathrm{a}}$, Jiaming GU ${ }^{\mathrm{b}}$, Zhenhua YAN ${ }^{\mathrm{a}}$, \\ Xiaosong YU ${ }^{\mathrm{b}}$, Yongli $\mathrm{ZHAO}^{\mathrm{b}, 1}$ \\ ${ }^{a}$ State Grid Electric Power Technical Research Institute, Yinchuan 750001, China \\ ${ }^{\mathrm{b}}$ Beijing University of Posts and Telecommunications, Beijing, 100876, China
}

\begin{abstract}
Mixed-grid optical networks are in a migration state where fixed-grid and flex-grid optical networks coexist. To carry point-to-multipoint (P2MP) services in mixed-grid optical networks, routing and resource allocation (RRA) problems need to be solved. Once the RRA fails, services will be blocked and then influence quality of service. The minimized spectrum for satisfying the bandwidth request of services is called as a frequency block (FB). For a service, the total number of available FBs embodies the spectrum availability on a link. Because the fixed-grid and flex-grid links have different channel spacing, spectrum availability on fixed-grid and flex-grid links needs different evaluation method. We propose a RRA algorithm in mixed-grid optical networks for P2MP services by being aware of spectrum availability. The spectrum availability is evaluated according to fixedgrid and flex-grid constraints. Our proposed algorithm achieves the lower blocking probability (BP) than that of benchmark RRA algorithms according to simulation results.
\end{abstract}

Keywords. Mixed-grid optical network, point-to-multipoint, resource allocation, routing, spectrum availability

\section{Introduction}

With the popularity of point-to-multipoint (P2MP) services such as online conferencing and IPTV, the fixed-grid Wavelength Division Multiplexing optical network, which has a low spectrum resource utilization and lacks flexibility, is hard to carry the increasing network traffic. It is necessary for the optical network to migrate from fixed grid to flexible grid. To prevent a large amount of interference to the existing fixed-grid optical network, the fixed grid and the flexible grid are still in a state of coexistence [1]. Such optical network is called as mixed-grid optical networks. For P2MP services, the same data is propagated from one source to multiple destinations. It is important to select a routing which occupies less spectrum resources for P2MP services in mixedgrid optical networks.

\footnotetext{
${ }^{1}$ Corresponding Author, Yongli Zhao, Beijing University of Posts and Telecommunications, Beijing,
} 100876, China; E-mail: yonglizhao@bupt.edu.cn. 
Much research about technologies and algorithms to carry P2MP services in optical networks has been conducted. A P2MP architecture based on digital subcarrier multiplexing has been presented, which saves remarkable cost and makes the network it enables simpler [2]. The multicast performance over spectrum elastic optical networks has been analyzed and results show the flexible spectrum allocation in elastic optical networks provides lower blocking probability for multicast compared to the ITU-T grid-based optical networks [3]. In elastic optical network, the all-optical P2MP problem has been investigated by using subtree schemes. And the proposed subtree algorithms could effectively reduce the request blocking probability by using slightly more bandwidth-variable transponders (BVTs) and better utilization of the higher-level modulation formats [4]. Flexible features of flex-grid networks are exploited for the dynamic placement of controllers enabled by virtualization of controllers [5]. A new algorithm, called mixed-grid-aware dynamic resource allocation, is proposed to solve the route, spectrum, and modulation-format allocation problem in a mixed-grid network while considering interoperability constraints [6]. A layered graph based routing and spectrum assignment algorithm for multicast is proposed by considering distance-adaptive modulation in mixed-grid optical networks [7].

In this paper, the problem of routing and resource allocation (RRA) for P2MP services is solved in mixed-grid optical networks. The proposed algorithm is aware of spectrum availability during the process of RRA with distance-adaptive modulation formats. According to the simulation results, it achieves the lowest blocking probability compared with benchmark RRA algorithms.

\section{Problem Statement and Network Model}

In mixed-grid optical networks, given the network topology, the RRA problem for P2MP services can be divided into two independent problems, including the routing problem and the resource allocation problem. 1) The routing problem means that when the P2MP service arrives, the routing trees need to be calculated from the source node to all the destination nodes. If the routing tree passes through the fixed-grid node and the bandwidth requirement exceeds the wavelength interval, the P2MP service needs to be supplied by multiple routing trees. 2) Resource allocation problem means that sufficient spectrum resources need to be allocated for it according to the bandwidth requirements of the P2MP service. During the allocation process, certain constraints need to be followed, including spectrum consistency, spectrum adjacency, and spectrum non-overlapping. This paper aims to solve the RRA problem by being aware of available spectrum resources for P2MP services in mixed-grid optical networks by designing appropriate algorithms, thereby reducing the blocking ratio of networks and improving network performances.

Topology $G(\boldsymbol{V}, \boldsymbol{E})$ for the physical network is given, where $\boldsymbol{V}=\left\{v_{1}, v_{2}, \ldots, v_{|\boldsymbol{V}|}\right\}$ and $\boldsymbol{E}=\left\{e_{1}, e_{2}, \ldots, e_{|E|}\right\}$ are the sets of fiber links and optical nodes. $e_{i}=\left(v_{s i}, v_{d i}, w\right)$, which is a three-tuple, starts from node $v_{s i}$ to node $v_{d i}$, and its weight equals $w . s_{e}=$ $\left\{f_{1}, f_{2}, \ldots, f_{F}\right\}$ is the bit-mask set to show status of frequency slots (FSs) on link e. $f_{i}=$ 0 if the $i^{\text {th }}$ FS is free, otherwise $f_{i}=1 ; F$ is the total number of FSs on link $e$. Two types of optical nodes including fixed-grid nodes and flex-grid nodes exist in the network. Link $e_{i}=\left(v_{s i}, v_{d i}, w\right)$ is a fixed-grid link when $v_{s i}, v_{d i}$ contains fixed-grid nodes; otherwise, it is a flex-grid link. A P2MP service is represented as $\operatorname{MR}(s, \boldsymbol{D}, b)$, 
where $s$ and $\boldsymbol{D}=\left\{d_{1}, d_{2}, \ldots, d_{|\boldsymbol{D}|}\right\}$ are the source node and the set of destinations respectively, $b$ is the bandwidth requirement. The minimized spectrum for satisfying the bandwidth request of services is called as a frequency block (FB). A FB can consist of several continuous FSs.

\section{Spectrum Availability Aware Routing and Resource Allocation (SAA-RRA) Algorithm in Mixed-Grid Optical Networks}

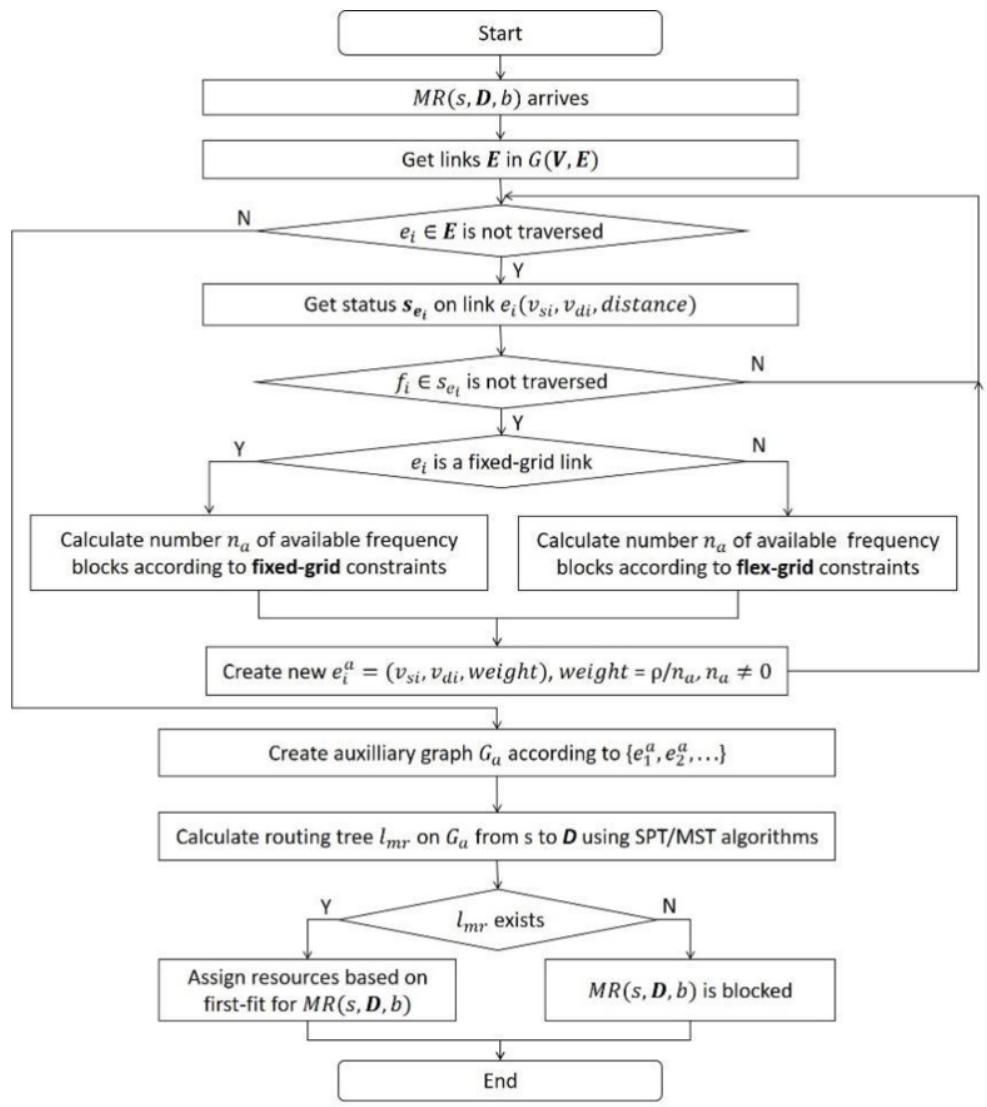

Figure 1. The flowchart of $S A A-R R A$ algorithm

For satisfying the requirement of P2MP services efficiently, we propose a spectrum availability aware routing and resource allocation $(S A A-R R A)$ algorithm in mixed-grid optical networks. The detailed flowchart of the proposed $S A A-R R A$ algorithm is shown in Figure 1. When a P2MP service $M R(s, \boldsymbol{D}, b)$ arrives, firstly the link set $\boldsymbol{E}$ is got. The proposed algorithm traverses $\boldsymbol{E}$ in network topology $G(\boldsymbol{V}, \boldsymbol{E})$. For the link $e_{i}=$ $\left(v_{s}, v_{d}\right.$, distance $) \in \boldsymbol{E}$, the bit-mask set $s_{e}$ is got which shows status of FSs on link $e_{i}$. Then, it traverses $f_{i}$ on $e_{i}$ to check whether the FS $f_{i}$ is free. During the FS status traversing process, the important step is to calculate the number of available FBs over different grid of links for the service $\operatorname{MR}(s, \boldsymbol{D}, b)$. If the $e_{i}$ is from flex-grid node to flex-grid node, the $e_{i}$ is a flex-grid link; otherwise, it is a fixed-grid link. If the $e_{i}$ is a fixed-grid link, the number of available FBs will be calculated according to fixed-grid 
constraints. And if the $e_{i}$ is a flex-grid link, the number of available FBs will be calculated according to flex-grid constraints.

Based on the calculated number $n_{a}$ of the available FBs over $e_{i}$, if $n_{a} \neq 0$, that is, available spectrums exist that meet the P2MP service's requirement, the new auxiliary link $e_{i}^{a}=\left(v_{s i}, v_{d i}\right.$, weight $)$ is created. Here, the source and destination of $e_{i}^{a}$ are the same with that of $e_{i}$ but the weight $=\rho / n_{a}$ is different, $\rho$ is a constant. The weight shows the number of available spectrum resources of the physical link, and the more the spectrum resources are available, the smaller the weight is. After traversing $\boldsymbol{E}$ in network topology $G(\boldsymbol{V}, \boldsymbol{E})$, the auxiliary graph $G_{a}\left(V_{a}, E_{a}\right)$ is created according to the established auxiliary links $e_{i}^{a}$. Then, routing tree is calculated on $G_{a}$ for $M R(s, \boldsymbol{D}, b)$ from source node $s$ to destination nodes $\boldsymbol{D}$ using minimum spanning tree (MST) and shortest path tree (SPT) algorithms. MST aims to reduce the total resource consumption. $S P T$ aims to reduce the time delay of data in the network. At last, if the calculated routing tree from source node to destination nodes exists, enough spectrum resources are assigned for $M R$ with First-fit and it means that the $M R$ is carried successfully; otherwise, the $M R$ is blocked.

\section{Simulation Results and Analysis}

The proposed $S A A-R A A$ algorithm is simulated with different routing tree calculation method $M S P$ and $S P T$ algorithms respectively, and are represented by $S A A-R R A-M S T$ and $S A A-R R A-S P T$. The compared RRA algorithms for P2MP are based on distanceaware RRA $(D A-R R A)$ algorithm. The benchmark algorithm firstly finds whether each link contains available FBs, and then compose the auxiliary topology of links with available FBs, and calculate the shortest path for the service according to the distance. The weight of link in the auxiliary topology is set to the distance of the corresponding physical link. $D A-R R A-M S T$ and $D A-R R A-S P T$ represent the compared algorithms that use $M S T$ and $S P T$ respectively. COST239 network topology is used in the simulation and it contains 11 nodes and 25 bi-directional links. The simulated traffic load is from

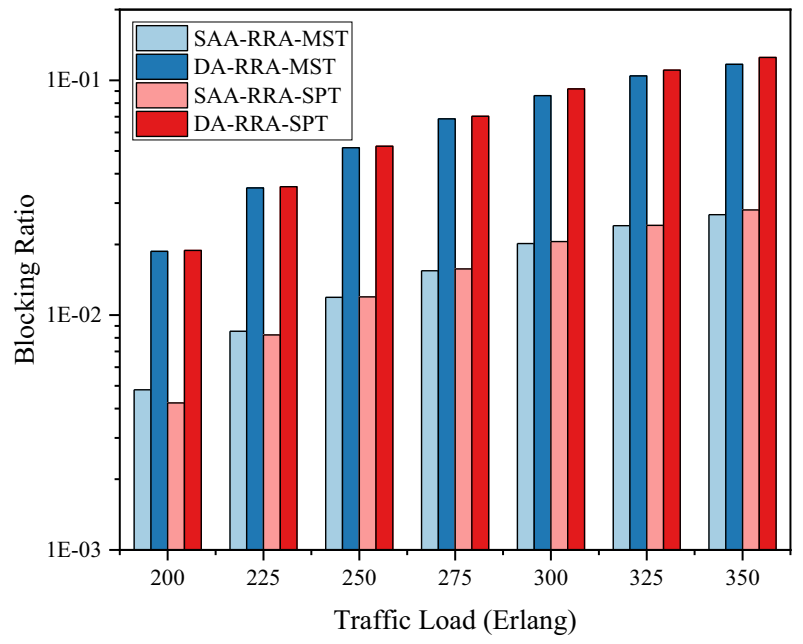

Figure 2. Simulation results in terms of blocking probability under 3 flex-grid nodes 
200 Erlang to 350 Erlang, and the spacing is 25 Erlang. We do the simulations under three different scenarios including different scenarios considering different number of flex-grid nodes in the network. In detail, simulations with flex-grid node set $\{2,4,6\}$, $\{2,4,6,8,10\},\{2,3,4,6,7,8,10,11\}$ are conducted respectively.

Figure 2 shows the BP of the simulated algorithms under 3 flex-grid nodes in mixed-grid optical network. We can find that SAA-RRA-MST and SAA-RRA-SPT algorithm have lower blocking probability (BP) in this case, because our proposed algorithm can be aware of available spectrum that meets the P2MP service's requirement by setting the weight of links in the constructed auxiliary graph based on the number of available FBs for services, while the benchmark algorithms cannot.

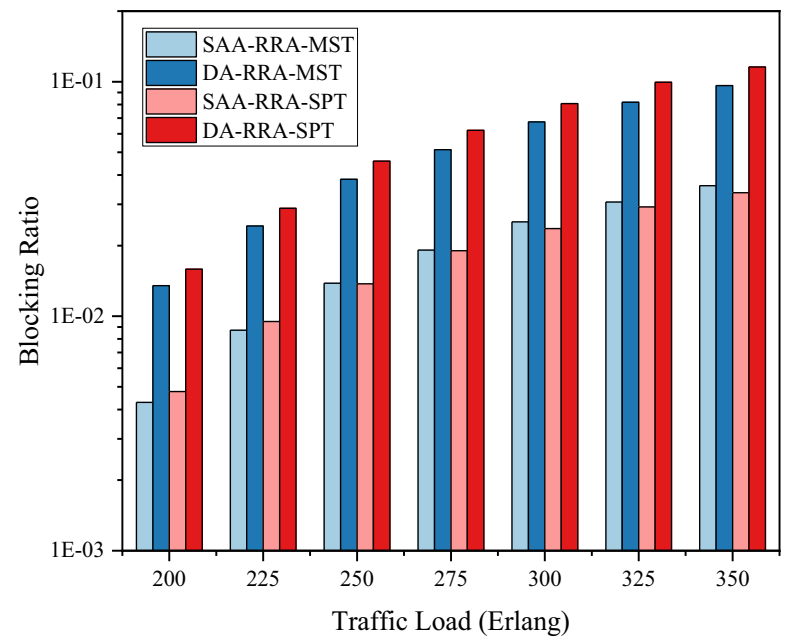

Figure 3. Simulation results in terms of blocking probability versus under 5 flex-grid nodes

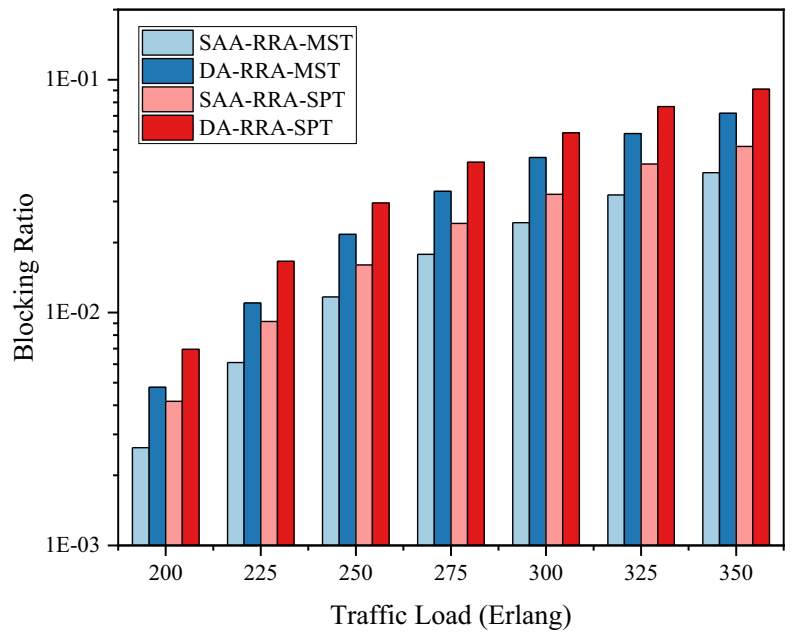

Figure 4. Simulation results in terms of blocking probability versus under 8 flex-grid nodes

Figure 3 shows the BP of some algorithms under 5 flex-grid nodes. The BP of our proposed algorithms is also the lowest in this case. The phenomenon shows that 
different numbers of flex-grid nodes in the network influence the gap between the $S A A$ $R R A$ and $D A-R R A$ algorithms. The reason is that the spectrum granularity of flex-grid link is smaller than that of flex-grid link, the same spectrum can carry more services. Hence, when flex-grid nodes increase, spectrum utilization of network is improved and the BP drops, and the benchmark algorithm is less likely to block the service due to the path selection.

Figure 4 shows the BP of some algorithms under 8 flex-grid nodes. The BP of our proposed algorithms is also the lowest. In this case, compared with Figure 2 and Figure 3 , the blocking probability of the $S A A-R R A(D A-R A A)$ algorithms by using $S P T$ and $M S T$ has a bigger gap. The reason is that the routing tree calculated by MST has the lower total weights compared with that calculated by SPT algorithms.

\section{Conclusion}

To solve the RRA in mixed-grid optical networks, we propose an algorithm called $S A A-R R A$ for P2MP services by being aware of spectrum availability, which is simulated with both $M S T$ and $S P T$ algorithm. Compared with the benchmark algorithm, the $S A A-R R A$ has lower BP. Simulation results show that the $S A A-R R A$ algorithms achieve the lowest blocking probability compared with benchmark algorithms. In cases where flex-grid nodes in physical topologies are different, the more flex-grid nodes, the more obvious the performance advantage of the $S A A-R A A$ algorithm.

\section{Acknowledgements}

This work was supported by fund of science and technology research and development project of Yinchuan (2021BDE13001).

\section{References}

[1] X. Yu et al. Migration from fixed grid to flexible grid in optical networks. IEEE Communications Magazine. 2015 Feb;53(2):34-43

[2] D. Welch et al. Point-to-Multipoint Optical Networks Using Coherent Digital Subcarriers. Journal of Lightwave Technology. 2021 Aug;39(16):5232-5247

[3] Q. Wang and L. Chen. Performance analysis of multicast traffic over spectrum elastic optical networks. OFC/NFOEC; 2012; pp. 1-3.

[4] Z. Fan, Y. Li, G. Shen and C. C. Chan. Distance-Adaptive Spectrum Resource Allocation Using Subtree Scheme for All-Optical Multicasting in Elastic Optical Networks. Journal of Lightwave Technology. 2017 May;35(9):1460-1468

[5] S. Rahman et al. Dynamic Controller Deployment for Mixed-Grid Optical Networks. 2018 Asia Communications and Photonics Conference (ACP); 2018; pp. 1-2.

[6] T. Ahmed et al. Dynamic routing, spectrum, and modulation-format allocation in mixed-grid optical networks. IEEE/OSA Journal of Optical Communications and Networking. 2020 May; 12(5):79-88.

[7] Q. Zhu, X. Yu, Y. Zhao and J. Zhang. Layered Graph based Routing and Spectrum Assignment for Multicast in Fixed/Flex-grid Optical Networks. 2020 Asia Communications and Photonics Conference (ACP) and International Conference on Information Photonics and Optical Communications (IPOC); 2020; pp. 1-3. 